\title{
Global Stabilization for Systems Evolving on Manifolds *
}

\author{
Michael Malisoff \\ Mikhail Krichman \\ Department of Mathematics \\ ALPHATECH, Inc. \\ Louisiana State University $\quad 6$ New England Executive Park \\ Baton Rouge, LA 70803 USA Burlington, MA 01803 USA \\ Eduardo Sontag \\ Department of Mathematics \\ Rutgers University \\ Hill Center-Busch Campus \\ New Brunswick, NJ 08903 USA
}

*MSC: 93B05. Running Head: Global Stabilization for Systems Evolving on Manifolds. Contact Author: Michael Malisoff; malisoff@1su.edu; Tel: (225) 578-1665; Fax: (225) 578-4276. 


\begin{abstract}
We show that any globally asymptotically controllable system on any smooth manifold can be globally stabilized by a state feedback. Since we allow discontinuous feedbacks, we interpret the solutions of our systems in the "sample and hold" sense introduced by Clarke-Ledyaev-Sontag-Subbotin (CLSS). Our work generalizes the CLSS Theorem which is the special case of our result for systems on Euclidean space. We apply our result to the input-to-state stabilization of systems on manifolds relative to actuator errors, under small observation noise.
\end{abstract}

Key Words: Asymptotic controllability, control systems on manifolds, input-to-state stabilization

\title{
1 Introduction
}

This note is devoted to the study of fully nonlinear systems

$$
\dot{x}=f(x, u), \quad x \in \mathcal{X}, \quad u \in \mathbf{U}
$$

evolving on arbitrary smooth manifolds $\mathcal{X}$ with inputs $u$ in general locally compact metric spaces $\mathbf{U}$, where $f$ is locally Lipschitz in $x$ uniformly for $u$ in compact sets, and jointly continuous in $(x, u)$. We assume that (1) is globally asymptotically controllable (GAC) to a given compact weakly invariant nonempty set $\mathcal{A} \subseteq \mathcal{X}$; see Section 3 below for the definition of GAC for systems on manifolds.

It is natural to inquire about the relationship between the GAC property for (1) and the existence of a feedback $k(x)$ such that the closed-loop system

$$
\dot{x}=f(x, k(x)), \quad x \in \mathcal{X}
$$

is globally asymptotically stable to $\mathcal{A}$. For the special case where the system (1) evolves on $\mathcal{X}=\mathbf{R}^{n}$ and $\mathcal{A}=\{0\}$, this relationship has been well studied (see [5] $8,10,11] 18]$ ). For that case, it is now well known that (1) does not in general admit a continuous stabilizing $k(x)$ (see [18, 19]). This negative result can also be seen from Brockett's Criterion (see [5, 16]) which states that a necessary condition for the existence of a continuous stabilizing feedback $k(x)$ for (1) with $k(0)=0$ is that $(x, u) \mapsto f(x, u)$ be open at zero; see also [15] pp. 252255] for a simple direct proof of Brockett's result using a homotopy. As a consequence, no totally nonholonomic 
mechanical system $\dot{x}=g_{1}(x) u_{1}+\ldots+g_{m}(x) u_{m}$ on $\mathbf{R}^{n}$ with $m<n$ and $\operatorname{rank}\left[g_{1}(0), \ldots, g_{m}(0)\right]=m$ is stabilizable by a continuous state feedback (see [16]). On the other hand, if (1) is GAC to $\mathcal{A}=\{0\}$ on $\mathbf{R}^{n}$, then it can be stabilized by a continuous time varying feedback $u=k(t, x)$ provided (i) the system is completely controllable with no drift or (ii) $n=1$ (see [8, 18] and Remark 6.4] below).

However, if we allow discontinuous feedbacks, then we have the following positive result from [7] known as the Clarke-Ledyaev-Sontag-Subbotin (CLSS) Theorem: If (1) is GAC to $\mathcal{A}$ on $\mathcal{X}=\mathbf{R}^{n}$, then there exists a discontinuous feedback $k(x)$ for which (2) is globally asymptotically stable to $\mathcal{A}$. Here and in the sequel, 'discontinuous' means 'not necessarily continuous in the state variable'. The discontinuous feedback $k(x)$ produces a discontinuous right-hand side in (2), which requires a more general interpretation of solutions that can be applied to discontinuous dynamics. In [7], this issue is resolved by interpreting the trajectories of [2] as "sample and hold" (a.k.a. CLSS) solutions (see Definition 2.4 below). The CLSS solution concept has been used extensively in nonlinear control analysis and controller design including the input-to-state stabilization of systems relative to actuator errors under small observation noise (see [10 11 [16] and Section 6 below). For example, CLSS solutions have been used to stabilize nonholonomic systems such as Brockett's Example which are not stabilizable by continuous state feedbacks (see [10]11]).

On the other hand, many important GAC systems evolve on manifolds other than $\mathbf{R}^{n}$ (e.g., stabilization of rigid bodies on the Lie group of rotations $\mathrm{SO}_{3}$ ) and are therefore not tractable by the CLSS Theorem. In fact, if (1) is GAC to a singleton $\mathcal{A}=\{p\}$ and admits a continuous stabilizing feedback $k(x)$, then a theorem of Milnor (see [12]) implies that $\mathcal{X}$ is diffeomorphic to Euclidean space. This is because the existence of $k(x)$ would imply the existence of a smooth control-Lyapunov function on $\mathcal{X}$ that could be viewed as a Morse function with a unique (possibly degenerate) critical point, and manifolds admitting such a Morse function are diffeomorphic to Euclidean space (see [16]). Therefore, even if (1) is holonomic, there may still be topological obstacles to continuous global stabilization when $\mathcal{X} \neq \mathbf{R}^{n}$.

Motivated by these considerations, this note will extend the CLSS Theorem to GAC systems on general smooth manifolds $\mathcal{X}$, proving the existence of a discontinuous feedback $k(x)$ rendering (2) globally stable to $\mathcal{A}$ in the sense of CLSS solutions. We follow the construction proposed in [17] which can be summarized as follows. We first 
embed $\mathcal{X}$ as a closed submanifold $g(\mathcal{X})$ of a Eucldean space $\mathbf{R}^{k}$ for some $k$, e.g., using the Whitney Embedding Theorem. Then we extend the system to all of $\mathbf{R}^{k}$ in such a way that (a) points outside $g(\mathcal{X})$ can be controlled to a tubular neighborhood of $\mathcal{A}$ and (b) $g(\mathcal{X})$ is invariant for the extended system. We then apply the CLSS Theorem to the extended system on $\mathbf{R}^{k}$ to design our feedback $k(x)$. The restriction of this feedback to $g(\mathcal{X})$ provides the desired stabilizer for the original system.

This note is organized as follows. In Section 2 we review CLSS solutions and the CLSS Theorem. We introduce the relevant definitions for stability on manifolds in Section 3 In Section 4 we prove our Generalized CLSS Theorem on the discontinuous stabilization of (1) on smooth manifolds. We illustrate our discontinuous feedback constructions in Section 5 We close in Section 6 by applying our results to the input-to-state stabilization of GAC systems on Riemannian manifolds relative to actuator errors under small observation noise. This extends the corresponding results [10,11] 14] on input-to-state stabilization for systems evolving on Euclidean space.

\section{CLSS Theorem on Euclidean Space}

In this section, we review the main definitions and results from [7] on the stabilization of GAC systems on Euclidean space. Throughout this section, our state space is $\mathcal{X}=\mathbf{R}^{n}$. We extend this material to systems on smooth manifolds in the next sections. We consider a system (1) for which $f$ is locally Lipschitz in $x$ uniformly for $u \in \mathbf{U}$ in compact sets, and jointly continuous in $x$ and $u$. Our input set $\mathbf{U}$ is a locally compact metric space with a metric $d_{\mathbf{U}}$ and a distinguished element $0 \in \mathbf{U}$, and we set $|u|=d_{\mathbf{U}}(u, 0)$ for each $u \in \mathbf{U}$. We let $\mathcal{U}$ denote the set of all

controls for (1), i.e., the set of all measurable, locally essentially bounded functions $\mathbf{u}: \mathbf{R}_{\geq 0} \rightarrow \mathbf{U}$. The essential supremum of any control $\mathbf{u} \in \mathcal{U}$ is denoted by $\|\mathbf{u}\|$, and

$$
\mathcal{U}_{N}=\{\mathbf{u} \in \mathcal{U}:\|\mathbf{u}\| \leq N\}
$$

for each $N>0$. Given $\xi \in \mathcal{X}$ and $\mathbf{u} \in \mathcal{U}$, the maximal trajectory of $1 \mathbf{1}$ for the control $\mathbf{u}$ that satisfies $x(0)=\xi$ is denoted by $x(t, \xi, \mathbf{u})$ or simply by $x(t)$ when $\xi$ and $\mathbf{u}$ are clear. We say that $x(t)$ is well defined provided it is defined for all $t \in \mathbf{R}_{\geq 0}:=[0, \infty)$. 
Let $\mathcal{A} \subseteq \mathcal{X}$. We say that $\mathcal{A}$ is weakly invariant (for $(\mathbb{D})$ provided there exists $N>0$ such that for any $\xi \in \mathcal{A}$ there is a control $\mathbf{u} \in \mathcal{U}_{N}$ such that the corresponding trajectory $x(t, \xi, \mathbf{u})$ is well defined and stays in $\mathcal{A}$. For example, $\mathcal{A}=\{0\}$ is weakly invariant if $f(0, \bar{a})=0$ for some $\bar{a} \in \mathbf{U}$. More generally, $\mathcal{A}$ could be a periodic orbit we wish to stabilize. We let $|p|$ denote the Euclidean norm of any $p \in \mathcal{X}$. We let bd (resp., clos) denote the boundary (resp., closure) operator, and we define the $\operatorname{distance} \operatorname{dist}(\mathcal{N}, x)=\inf \{|p-x|: p \in \mathcal{N}\}$ for any subset $\mathcal{N} \subseteq \mathbf{R}^{n}$ and $x \in \mathbf{R}^{n}$. For any $x \in \mathcal{X}$, we let $|x|_{\mathcal{A}}$ denote the distance from $x$ to $\mathcal{A}$. Therefore, $|x|_{\mathcal{A}}<\varepsilon$ means $x \in \mathcal{B}_{\varepsilon}(\mathcal{A}):=\left\{p \in \mathbf{R}^{n}: \operatorname{dist}(\mathcal{A}, p)<\varepsilon\right\}$.

We next state two equivalent definitions of globally asymptotic controllability. First we state the well known definition from [10, 16] in terms of comparison functions. We then provide the original $\varepsilon-\delta$ formulation which we generalize to systems on manifolds in the next section. We use the following comparison function definitions from [16]. A function $\alpha: \mathbf{R}_{\geq 0} \rightarrow \mathbf{R}_{\geq 0}$ is said to be of class $\mathcal{K}$ provided $\alpha$ is continuous, strictly increasing, and satisfies $\alpha(0)=0$; it is of class $\mathcal{K}_{\infty}$ provided it is also unbounded. We say $\alpha: \mathbf{R}_{\geq 0} \rightarrow \mathbf{R}_{\geq 0}$ is of class $\mathcal{N}$ provided $\alpha$ is non-decreasing; and of class $\mathcal{L}$ provided $\alpha(s)$ is decreasing to 0 as $s \rightarrow+\infty$. A function $\beta: \mathbf{R}_{\geq 0} \times \mathbf{R}_{\geq 0} \rightarrow \mathbf{R}_{\geq 0}$ is said to be of class $\mathcal{K} \mathcal{L}$ provided (a) $\beta(s, \cdot) \in \mathcal{L}$ for every fixed $s$ and (b) $\beta(\cdot, t) \in \mathcal{K}$ for every fixed $t$. We write $\beta \in \mathcal{K} \mathcal{L}$ to mean that $\beta$ is of class $\mathcal{K} \mathcal{L}$ and similarly for the other types of comparison functions.

Definition 2.1 Let $\mathcal{A} \subseteq \mathcal{X}$ be compact, nonempty, and weakly invariant for (1). We call (1) globally asymptotically controllable (GAC) to $\mathcal{A}$ (on $\mathcal{X}$ ) provided there exist $\beta \in \mathcal{K} \mathcal{L}$ and $\sigma \in \mathcal{N}$ such that for each $\xi \in \mathcal{X}$, there exists a control $\mathbf{u}$ with $\|\mathbf{u}\| \leq \sigma\left(|\xi|_{\mathcal{A}}\right)$ such that $x(t, \xi, \mathbf{u})$ is well defined and satisfies $|x(t, \xi, \mathbf{u})|_{\mathcal{A}} \leq \beta\left(|\xi|_{\mathcal{A}}, t\right)$ for all $t \geq 0$.

The following equivalent formulation of GAC has a natural generalization to systems on manifolds; see Definition 3.1] below. See [1] for the equivalence of our GAC definitions on $\mathbf{R}^{n}$.

Definition 2.2 Let $\mathcal{A} \subseteq \mathcal{X}$ be compact, nonempty, and weakly invariant for (1). We call (1) globally asymptotically controllable $(G A C)$ to $\mathcal{A}$ (on $\mathcal{X}$ ) provided for all $\varepsilon_{1}, \varepsilon_{2}>0$ with $\varepsilon_{1}<\varepsilon_{2}$, we have:

1. There exist $T=T\left(\varepsilon_{1}, \varepsilon_{2}\right)>0$ and $\delta=\delta\left(\varepsilon_{1}\right)>0$ such that for each $\xi \in \mathcal{B}_{\varepsilon_{2}}(\mathcal{A})$, there exists a control $\mathbf{u}$ such that 
(a) $x(t, \xi, \mathbf{u})$ is well defined;

(b) $x(t, \xi, \mathbf{u}) \in \mathcal{B}_{\varepsilon_{1}}(\mathcal{A})$ for all $t>T$; and

(c) if also $\xi \in \mathcal{B}_{\delta}(\mathcal{A})$, then $\mathbf{u}$ can be chosen so that $x(t, \xi, \mathbf{u}) \in \mathcal{B}_{\varepsilon_{1}}(\mathcal{A})$ for all $t \geq 0$.

2. For every positive number $\varepsilon<\varepsilon_{2}$, there exists $N=N(\varepsilon)>0$ such that if $\xi$ from 1 . also satisfies $\xi \in \mathcal{B}_{\varepsilon}(\mathcal{A})$, then the control $\mathbf{u}$ from 1 . can be chosen with $\mathbf{u} \in \mathcal{U}_{N}$.

Definition 2.3 A feedback for (1) is defined to be any locally bounded function $k: \mathcal{X} \rightarrow \mathbf{U}$.

In this note, we study the equivalence of (open loop) asymptotic controllability of 1 and the possibility of stabilizing the system to a weakly invariant set $\mathcal{A}$ via a state feedback. The novelty of our work lies in its applicability to systems on general smooth manifolds. Even for systems on $\mathbf{R}^{n}$, it is often the case that a continuous stabilizing state feedback does not exist (see [10 11] 16]). However, a discontinuous feedback is always possible to construct, provided we use the Clarke-Ledyaev-Sontag-Subbotin (CLSS) definition of a "sample and hold" solution for a discontinuous dynamic. We review this generalized solution notion next, following the notation from [10. 11 .

We define a partition ( of $\mathbf{R}_{\geq 0}$ ) to be any divergent sequence $\pi: 0=t_{0}<t_{1}<t_{2}<\ldots$ and we call

$$
\overline{\mathbf{d}}(\pi)=\sup _{i \geq 0}\left(t_{i+1}-t_{i}\right) \quad\left(\operatorname{resp} ., \underline{\mathbf{d}}(\pi)=\inf _{i \geq 0}\left(t_{i+1}-t_{i}\right)\right)
$$

the upper (resp., lower) diameter of the partition $\pi=\left\{t_{o}, t_{1}, t_{2}, \ldots\right\}$.

Definition 2.4 Let $k$ be a feedback for the system 1 , $\xi \in \mathcal{X}$, and $\pi=\left\{t_{i}\right\}_{i \geq 0}$ be a partition. The $\pi$-trajectory

$$
t \mapsto x_{\pi}(t, \xi, k)
$$

for 11, $\xi, \pi$, and $k$ is defined to be the continuous function obtained by recursively solving

$$
\dot{x}(t)=f\left(x(t), k\left(x\left(t_{i}\right)\right)\right)
$$

from the initial time $t_{i}$ up to the maximal time

$$
s_{i}=\max \left\{t_{i}, \sup \left\{s \in\left[t_{i}, t_{i+1}\right]: x(\cdot) \text { is defined on }\left[t_{i}, s\right)\right\}\right\},
$$


where $x(0)=\xi \cdot{ }^{1}$ The domain of $x_{\pi}(\cdot, \xi, k)$ is $\left[0, t_{\max }\right)$, where

$$
t_{\max }=\inf \left\{s_{i}: s_{i}<t_{i+1}\right\}
$$

We call $x_{\pi}(\cdot, \xi, k)$ well defined provided $t_{\max }=+\infty$.

The $t_{i}$ argument in the maximum (3) is needed to allow the possibility that $x(\cdot)$ is not defined at all on $\left[t_{i}, t_{i+1}\right]$ in which case the supremum in (3) alone would by definition give $-\infty$. The following notion of (global) stabilization for (1) was introduced in [7]:

Definition 2.5 A feedback $k: \mathcal{X} \rightarrow \mathbf{U}$ is said to s-stabilize the system (1) to $\mathcal{A}$ provided for each pair $(r, R)$ with $0<r<R$ there exist $M=M(R)>0$ with $\lim _{R \rightarrow 0} M(R)=0, \delta=\delta(r, R)>0$, and $T=T(r, R)>0$ such that, for every $\pi$ with $\overline{\mathbf{d}}(\pi)<\delta$ and $\xi \in \mathcal{B}_{R}(\mathcal{A})$, the $\pi$-trajectory $x(\cdot)$ for $\mathbb{1}$, initial value $\xi$, partition $\pi$, and feedback $k$ is well defined and satisfies (a) $|x(t)|_{\mathcal{A}} \leq r$ for all $t \geq T$ and (b) $|x(t)|_{\mathcal{A}} \leq M(R)$ for all $t \geq 0$.

The following result to be generalized was shown in [7] for $\mathcal{A}=\{0\}$ but can be shown for our general compact, nonempty, weakly invariant set $\mathcal{A} \subseteq \mathcal{X}=\mathbf{R}^{n}$ by similar arguments (e.g., using the existence results from [9] for locally Lipschitz Lyapunov functions for GAC systems and any compact set $\mathcal{A}$ ):

Theorem 1 If (I) is GAC to $\mathcal{A}$ on $\mathcal{X}=\mathbf{R}^{n}$, then it admits a feedback that s-stabilizes the system to $\mathcal{A}$.

The preceding result is called the CLSS Theorem. Our main contribution is a Generalized CLSS Theorem for systems on smooth manifolds and is the subject of the next two sections. We provide related results on input-tostate stabilization on Riemannian manifolds in Section 6

\section{Stabilization on Manifolds}

We again consider the system (1) but we assume from now on that the state space $\mathcal{X}$ for the system is an arbitrary smooth (i.e., $C^{\infty}$ ) (second countable) manifold. Controls $\mathbf{u}$, as before, are measurable, locally essentially bounded

${ }^{1}$ The continuity requirement for $x(\cdot)$ amounts to stipulating that the final value on the previous subinterval is used as the initial value at the next subinterval. 
functions $\mathbf{u}: \mathbf{R}_{\geq 0} \rightarrow \mathbf{U}$. We assume

$$
f: \mathcal{X} \times \mathbf{U} \rightarrow T_{x}(\mathcal{X}):(x, u) \mapsto f(x, u)
$$

is locally Lipschitz in $x$ and jointly continuous in $x$ and $u$; that is

$$
f(x, u)=\sum_{i} a_{i}(x, u) \frac{\partial}{\partial x_{i}}
$$

where each $a_{i}: \mathcal{X} \times \mathbf{U} \rightarrow \mathbf{R}$ is locally Lipschitz in $x$ uniformly for $u$ in compact sets and jointly continuous, and $T_{x}(\mathcal{X})$ is the tangent space to $\mathcal{X}$ at $x$. We define the solutions $x(t, \xi, \mathbf{u})$ of $\mathbb{1}$ as before. We next generalize Definition 2.2 for GAC to manifolds.

Let $\mathcal{A}$ be a compact, nonempty, weakly invariant subset of $\mathcal{X}$ for 1 , and let $\mathcal{P} \mathcal{N}^{\mathcal{A}}$ denote the set of all open precompact subsets of $\mathcal{X}$ containing $\mathcal{A}$. To extend the GAC definition to manifolds, we simply replace the $\varepsilon$-neighborhoods of $\mathcal{A}$ from Definition 2.2 with arbitrary sets in $\mathcal{P N} \mathcal{N}^{\mathcal{A}}$ as follows:

Definition 3.1 We say that 1 is globally asymptotically controllable $(G A C)$ to $\mathcal{A}$ (on $\mathcal{X}$ ) provided:

1. Given any $\mathcal{E}_{1}, \mathcal{E}_{2} \in \mathcal{P} \mathcal{N}^{\mathcal{A}}$ with $\mathcal{E}_{1} \subseteq \mathcal{E}_{2}$, there exist $T=T\left(\mathcal{E}_{1}, \mathcal{E}_{2}\right)>0$ and $\Delta=\Delta\left(\mathcal{E}_{1}\right) \in \mathcal{P} \mathcal{N}^{\mathcal{A}}$ such that for every $\xi \in \mathcal{E}_{2}$ there exists a control $\mathbf{u}$ such that

(a) $x(t, \xi, \mathbf{u})$ is well defined;

(b) $x(t, \xi, \mathbf{u}) \in \mathcal{E}_{1}$ for all $t>T$; and

(c) if also $\xi \in \Delta$, then $\mathbf{u}$ can be chosen so that $x(t, \xi, \mathbf{u}) \in \mathcal{E}_{1}$ for all $t \geq 0$.

2. For every set $\mathcal{N} \in \mathcal{P} \mathcal{N}^{\mathcal{A}}$, there exists $N=N(\mathcal{N})>0$ such that if $\xi$ from 1. also satisfies $\xi \in \mathcal{N}$, then the control $\mathbf{u}$ from 1. can be chosen with $\mathbf{u} \in \mathcal{U}_{N}$.

We assume throughout this section that our dynamic $f$ is GAC to $\mathcal{A}$. Since our definitions of feedback and $\pi$-trajectory from Section 2 do not depend on the structure of the state space $\mathcal{X}$, they remain valid for systems on manifolds. We extend the definition of an s-stabilizing feedback to manifolds as follows:

Definition 3.2 A feedback $k: \mathcal{X} \rightarrow \mathbf{U}$ is said to s-stabilize the system (1) to $\mathcal{A}$ provided the following hold for all sets $\mathcal{R}_{1}, \mathcal{R}_{2} \in \mathcal{P} \mathcal{N}^{\mathcal{A}}$ with $\mathcal{R}_{1} \subseteq \mathcal{R}_{2}$ : 
1. There exist a set $\mathcal{M}=\mathcal{M}\left(\mathcal{R}_{2}\right) \subseteq \mathcal{X}$ and numbers $\delta=\delta\left(\mathcal{R}_{1}, \mathcal{R}_{2}\right)>0$ and $T=T\left(\mathcal{R}_{1}, \mathcal{R}_{2}\right)$ such that, for any partition $\pi$ with $\overline{\mathbf{d}}(\pi)<\delta$ and any $\xi$ in $\mathcal{R}_{2}$, the $\pi$-trajectory $x(\cdot)$ for $(\mathbb{1}$, the initial state $\xi$, and the feedback $k$ is well defined and satisfies: (a) $x(t) \in \mathcal{R}_{1}$ for all $t \geq T$ and (b) $x(t) \in \mathcal{M}$ for all $t \geq 0$.

2. For each set $\mathcal{E} \in \mathcal{P} \mathcal{N}^{\mathcal{A}}$ there exists $\mathcal{D} \in \mathcal{P} \mathcal{N}^{\mathcal{A}}$ such that if $\mathcal{R}_{2} \subseteq \mathcal{D}$, then the set $\mathcal{M}$ in 1 . can be chosen so that $\mathcal{M} \subseteq \mathcal{E}$.

Our goal is to show that the CLSS Theorem remains true on any smooth manifold $\mathcal{X}$. To this end, we follow the strategy outlined in [17] which can be summarized as follows. We first embed the state space manifold $\mathcal{X}$ into some Euclidean space $\mathbf{R}^{k}$ (e.g., using the Whitney Embedding Theorem). Then we extend the dynamic to all of $\mathbf{R}^{k}$ in such a way that (a) the system is asymptotically controllable to a tubular neighborhood of $\mathcal{A}$ and (b) $\mathcal{X}$ is a strongly invariant set under the extended system (see Lemma 3.5. Next we apply the CLSS Theorem to the extended system. Thus, we get an s-stabilizing feedback on $\mathbf{R}^{k}$. When restricted to $\mathcal{X}$, this feedback will s-stabilize (1) to $\mathcal{A}$.

To make this construction precise, we use the following definitions and facts from differential topology (see [3, 4]). The following is known as the Whitney Embedding Theorem (see [4] p.92]):

Lemma 3.3 If $\mathcal{X}$ is an $n$-dimensional smooth manifold, then there exists an embedding $g: \mathcal{X} \rightarrow \mathbf{R}^{2 n+1}$ for which $g(\mathcal{X})$ is a submanifold, and a closed subset, of $\mathbf{R}^{2 n+1}$.

By Lemma 3.3 we can assume that our state space $\mathcal{X}$ is a smooth submanifold of $\mathbf{R}^{k}$ with $\mathcal{X} \subseteq \mathbf{R}^{k}$ closed. The normal bundle $\Xi(\mathcal{X})$ of $\mathcal{X}$ in $\mathbf{R}^{k}$ is defined by

$$
\Xi(\mathcal{X})=\left\{\langle x, \mathrm{q}\rangle \in \mathcal{X} \times \mathbf{R}^{k}: \mathrm{q} \perp T_{x}(\mathcal{X})\right\}
$$

We define the projections $\pi_{M}: \Xi(\mathcal{X}) \rightarrow \mathcal{X}$ by $\pi_{M}(\langle x, \mathrm{q}\rangle)=x$ and $\pi_{N}: \Xi(\mathcal{X}) \rightarrow \mathbf{R}^{k}$ by $\pi_{N}(\langle x, \mathrm{q}\rangle)=\mathrm{q}$, and $\theta(\langle x, q\rangle):=x+q$. For each smooth function $\omega: \mathcal{X} \rightarrow \mathbf{R}_{>0}$, the $\omega$-tube $\Xi(\mathcal{X}, \omega)$ is defined by

$$
\Xi(\mathcal{X}, \omega)=\{\langle x, \mathrm{q}\rangle \in \Xi(\mathcal{X}):|\mathrm{q}|<\omega(x)\}
$$

The next result is known as the Tubular Neighborhood Theorem. 
Lemma 3.4 Let $\mathcal{X}$ be a closed submanifold of $\mathbf{R}^{k}$. There exists a smooth function $\omega: \mathcal{X} \rightarrow \mathbf{R}_{>0}$ such that $\theta: \Xi(\mathcal{X}, \omega) \rightarrow \mathbf{R}^{k}:\langle x, \mathrm{q}\rangle \mapsto x+\mathrm{q}$ is a diffeomorphism onto an open neighborhood of $\mathcal{X}$ in $\mathbf{R}^{k}$.

In particular, $\Xi(\mathcal{X}, \omega)$ is an open subset of $\mathbf{R}^{k} \times \mathbf{R}^{k}$. Pick functions $\omega$ and $\theta$ as in Lemma 3.4 for our state space manifold $\mathcal{X}$. Since $\mathcal{A} \subseteq \mathcal{X}$ is compact and $\omega$ is continuous, $\omega$ attains its minimum on $\mathcal{A}$. Let

$$
\varepsilon=\frac{1}{2} \min _{x \in \mathcal{A}} \omega(x)
$$

and for each set $\mathcal{A}_{1} \subseteq \mathcal{X}$, define

$$
\begin{aligned}
& \Xi\left(\mathcal{A}_{1}, \varepsilon\right)=\left\{\langle x, \mathrm{q}\rangle \in \Xi(\mathcal{X}): x \in \mathcal{A}_{1},|\mathrm{q}|<\varepsilon\right\}, \\
& \Xi\left(\mathcal{A}_{1}, \omega\right)=\left\{\langle x, \mathrm{q}\rangle \in \Xi(\mathcal{X}): x \in \mathcal{A}_{1},|\mathrm{q}|<\omega(x)\right\}, \\
& \mathbf{T N}_{\varepsilon} \mathcal{A}_{1}=\theta\left(\Xi\left(\mathcal{A}_{1}, \varepsilon\right)\right), \quad \mathbf{T N}_{\omega} \mathcal{A}_{1}=\theta\left(\Xi\left(\mathcal{A}_{1}, \omega\right)\right) .
\end{aligned}
$$

Notice that if $\varepsilon \leq \omega(x)$ for all $x \in \mathcal{A}_{1}$, then $\mathbf{T N}_{\varepsilon} \mathcal{A}_{1} \subseteq \mathbf{T N}_{\omega} \mathcal{A}_{1} \subseteq \mathbf{T N}_{\omega} \mathcal{X}$. Also, clos $\mathbf{T} \mathbf{N}_{\varepsilon} \mathcal{A}$ is a compact subset of $\mathbf{T N}_{\omega} \mathcal{X}$. Next consider the system

$$
\dot{x}=f(x, u), \quad \dot{\mathrm{q}}=\mathrm{q} v, \quad\langle x, q\rangle \in \mathcal{X} \times \mathbf{R}^{k}, \quad\langle u, v\rangle \in \mathbf{U} \times \mathbf{R},
$$

whose (maximal) solution for the controls $\langle\mathbf{u}, \mathbf{v}\rangle$ starting at $\left\langle\xi, q_{0}\right\rangle$ we denote by $\left\langle x(t, \xi, \mathbf{u}), \mathrm{q}\left(t, q_{0}, \mathbf{v}\right)\right\rangle$, or by $\langle x(t), \mathrm{q}(t)\rangle$ for brevity. If, for some initial state $\langle x(0), \mathrm{q}(0)\rangle$ and controls $\langle\mathbf{u}, \mathbf{v}\rangle$, the trajectory $\langle x(t), \mathrm{q}(t)\rangle$ of $\mathbf{5}$ ) stays in $\Xi(\mathcal{X}, \omega)$, then $y\left(t, y_{0}, \mathbf{u}, \mathbf{v}\right)=\theta(\langle x(t), \mathrm{q}(t)\rangle)$ is the corresponding trajectory of

$$
\dot{y}=f_{1}(y, u, v):=f\left(\pi_{M}\left(\theta^{-1}(y)\right), u\right)+\pi_{N}\left(\theta^{-1}(y)\right) v, \quad y \in \mathbf{T N}_{\omega} \mathcal{X}, \quad\langle u, v\rangle \in \mathbf{U} \times \mathbf{R}
$$

with the initial value $y_{0}=y(0)=\theta(\langle x(0), \mathrm{q}(0)\rangle)$. We denote this solution by $y(t)$ when no confusion would result. We also omit the $\theta^{-1}$ inside the projections $\pi_{N}$ and $\pi_{M}$ in the sequel to simplify our notation. We (discontinuously) extend $f_{1}$ to $\mathbf{R}^{k}$ by defining it to be zero outside $\mathbf{T} \mathbf{N}_{\omega} \mathcal{X}$.

Next, we extend our GAC system (1) to all of $\mathbf{R}^{k}$ as follows. Let $\mathcal{X}^{\sharp} \subseteq \mathbf{R}^{k}$ be any closed set contained in $\mathbf{T N}_{\omega} \mathcal{X}$ and containing $\mathcal{X}$ in its interior. Let $C_{\omega} \subseteq \mathbf{R}^{k}$ be any open set such that the following holds:

$$
\mathbf{R}^{k} \backslash \mathbf{T N}_{\omega} \mathcal{X} \subseteq C_{\omega} \subseteq \operatorname{clos} C_{\omega} \subseteq \mathbf{R}^{k} \backslash \mathcal{X}^{\sharp} .
$$


Then $\operatorname{bd} C_{\omega} \subseteq \mathbf{T N}_{\omega} \mathcal{X}$. Let $\phi: \mathbf{R}^{k} \rightarrow[0,1]$ be any smooth function such that

$$
\phi(z)= \begin{cases}1 & z \in \mathcal{X}^{\sharp} \\ 0 & z \in \operatorname{clos} C_{\omega}\end{cases}
$$

which exists by a well known separation result (e.g., [3] Exercise V.4.5]). Now define a system

$$
\dot{z}=f_{2}(z, u, v, w):=f_{1}(z, u, v) \phi(z)+(1-\phi(z)) w, \quad z \in \mathbf{R}^{k}, \quad\langle u, v, w\rangle \in \mathbf{U} \times \mathbf{R} \times \mathbf{R}^{k},
$$

whose (maximal) solution starting at $z_{o}$ for given controls $\langle\mathbf{u}, \mathbf{v}, \mathbf{w}\rangle$ we denote by $z\left(t, z_{0}, \mathbf{u}, \mathbf{v}, \mathbf{w}\right)$. Since $\phi \equiv 0$ in $C_{\omega}$, we know $f_{2}$ is locally Lipschitz in $z \in \mathbf{R}^{k}$. We use the following elementary observation:

Lemma 3.5 Any trajectory $z(t)$ for $f_{2}$ starting at a point $\eta \in \mathcal{X}$ remains in $\mathcal{X}$ on its domain of definition and therefore is a trajectory of $f$. In other words, $\mathcal{X}$ is strongly invariant for $f_{2}$.

Proof. Since $\langle x, 0\rangle \in \Xi(\mathcal{X}, \omega)$ for all $x \in \mathcal{X}$, the uniqueness property for solutions of $\left[\right.$ in $\mathbf{T N}_{\omega} \mathcal{X}$ implies that all trajectories of $f_{1}$ starting in $\mathcal{X}$ remain in $\mathcal{X}$ and so are trajectories of $f$. On the other hand, trajectories $z(t)$ of $f_{2}$ starting in $\mathcal{X}$ are also trajectories of $f_{1}$ while they are in $\mathcal{X}^{\sharp}$ (by our choice $(7)$ of $\phi$ ), since $f_{1}$ and $f_{2}$ agree on $\mathcal{X}^{\sharp}$. By the uniqueness property for trajectories of $f_{1}, z(t)$ therefore cannot enter $\mathcal{X}^{\sharp} \backslash \mathcal{X} \subseteq \mathbf{T N}_{\omega} \mathcal{X}$ and so stays in $\mathcal{X}$. Hence $z(t)$ is a trajectory of $f_{1}$, and also for $f$.

The preceding lemma forms the basis for our Generalized CLSS Theorem in the next section.

\section{CLSS Theorem on Manifolds}

In this section, we prove the following Generalized CLSS Theorem for any smooth manifold $\mathcal{X}$ and any compact, nonempty, weakly invariant set $\mathcal{A} \subseteq \mathcal{X}$ for (1):

Theorem 2 If (Z) is GAC to $\mathcal{A}$ on the manifold $\mathcal{X}$, then it admits a feedback that s-stabilizes the system to $\mathcal{A}$.

This will follow from the following key lemma:

Lemma 4.1 If the system (11) is GAC to $\mathcal{A}$ on $\mathcal{X}$, then the system (8) is GAC to $\operatorname{clos} \mathbf{T} \mathbf{N}_{\varepsilon} \mathcal{A}$ on $\mathbf{R}^{k}$. 
We begin by proving Lemma 4.1] Fix $z_{0} \in \mathbf{R}^{k}$, a precompact open set $\mathcal{B}$ containing $\operatorname{clos} \mathbf{T N} \mathcal{A}_{\varepsilon} \mathcal{A}$, and an open set $\mathcal{A}_{1} \in \mathcal{P} \mathcal{N}^{\mathcal{A}}$ such that $\mathbf{T N}_{\varepsilon} \mathcal{A}_{1} \subseteq \mathcal{B}$. Assume first that $\eta:=z_{0} \in \mathbf{T N}_{\omega} \mathcal{X}$. Since $\mathbb{1}$ is GAC to $\mathcal{A}$, we can find a control $\mathbf{u}:[0, \infty) \rightarrow \mathbf{U}$ and constants $T_{1}>0$ and $p_{1}>0$ with $\|\mathbf{u}\|<p_{1}$ such that the trajectory $x(t)=x\left(t, \pi_{M}(\eta), \mathbf{u}\right)$ of 10 is well defined and satisfies $x(t) \in \mathcal{A}_{1}$ for all $t \geq T_{1}$. This gives a compact set $\bar{B} \subseteq \mathcal{X}$ containing $\mathcal{A}$ such that $x(t)=x\left(t, \pi_{M}(\eta), \mathbf{u}\right) \in \bar{B}$ for all $t \geq 0$.

Since $\omega$ is positive and smooth on $\mathcal{X}$, there exist positive values

$$
p_{2}=1+\max _{x \in \bar{B}}|\nabla \omega(x)|, \quad p_{4}=\min _{x \in \bar{B}} \omega(x)
$$

and $p_{3}>0$ such that $|f(x, u)|<p_{3}$ for all $x \in \bar{B}$ and $|u|<p_{1}$. Then $p_{4} / 2 \leq \varepsilon$, and

$$
\left|\frac{d}{d t} \omega(x(t))\right|=|\nabla \omega(x(t)) \cdot f(x(t), \mathbf{u}(t))| \leq p_{2} p_{3} \quad \text { for almost all } t \geq 0 .
$$

In other words, $p_{2} p_{3}$ is an upper bound on the rate of change of the width $\omega(x(t))$ of $\mathbf{T N}_{\omega} \mathcal{X}$, as we move along the trajectory $x(t)$. Hence, to ensure that our stabilizing trajectory of $(8)$ starting in $\mathbf{T N}_{\omega} \mathcal{X}$ stays there, we must design a control $\mathbf{v}$ so that the solution of $\left[\right.$ is pushed towards $\mathcal{X}$ faster than $p_{2} p_{3}$.

Since we assumed $\eta \in \mathbf{T N}_{\omega} \mathcal{X}$, we have $\left\langle\pi_{M}(\eta), \pi_{N}(\eta)\right\rangle \in \Xi(\mathcal{X}, \omega)$ and therefore $\left|\pi_{N}(\eta)\right|<\omega\left(\pi_{M}(\eta)\right)$. Define $\mathbf{v}:[0,+\infty) \rightarrow \mathbf{R}$ by

$$
\mathbf{v}(t)=\left\{\begin{array}{cc}
-\frac{p_{2} p_{3}}{p_{4} / 4} & t \in\left[0, T_{2}\right] \\
0 & t>T_{2}
\end{array} \quad, \quad \text { where } T_{2}=\max \left\{0, \frac{\left|\pi_{N}(\eta)\right|-p_{4} / 4}{p_{2} p_{3}}\right\}\right.
$$

Let $q(t)$ be the solution of $\dot{q}=\mathbf{v} q$ starting at $\pi_{N}(\eta)$. Set $y(t)=x(t)+q(t)$, where $x(t)=x\left(t, \pi_{M}(\eta), \mathbf{u}\right)$ is defined above; then $y(t)$ has domain $[0, \infty)$, and $\langle x(t), q(t)\rangle$ is a solution of (5) on $[0,+\infty)$. We next define $t^{\prime}=\inf \{t \geq 0:\langle x(t), q(t)\rangle \in \operatorname{bd} \Xi(\mathcal{X}, \omega)\}$, so $\langle x(t), q(t)\rangle \in \Xi(\mathcal{X}, \omega)$ on $\left[0, t^{\prime}\right)$. We show that $t^{\prime}=+\infty$. This will show that $y(t)$ is a solution of $(6)$ on all of $\mathbf{R}_{\geq 0}$. To this end, first note that:

(i) Since the direction of $\mathbf{v}(t) q(t)$ is always opposite to that of $q(t)$ whenever $\mathbf{v}(t) \neq 0$, the function $|q(t)|$ is non-increasing on $\mathbf{R}_{\geq 0}$.

(ii) At all points $t \in\left[0, T_{2}\right]$ for which $\dot{x}(t)$ exists and $|q(t)| \geq p_{4} / 4$, the following holds:

$$
\frac{d}{d t}|q(t)|=-\frac{p_{2} p_{3}}{p_{4} / 4}|q(t)| \leq-p_{2} p_{3} \leq-\left|\frac{d}{d t} \omega(x(t))\right| \leq \frac{d}{d t} \omega(x(t)) .
$$


By separately considering the case where $|q(t)|$ stays above $p_{4} / 4$ on $\left[0, T_{2}\right]$ and using (11)-(12), one can easily check that $\left|q\left(T_{2}\right)\right| \leq p_{4} / 4$; this inequality is clear if $|q(t)|$ ever goes below $p_{4} / 4$ on $\left[0, T_{2}\right]$, by (i). Hence, $|q(t)| \leq p_{4} / 4$ for all $t \geq T_{2}$, by the choice of $\mathbf{v}$. Similarly, we can use 112 , the definition of $p_{4}$, and the fact that $\left|\pi_{N}(\eta)\right|<\omega\left(\pi_{M}(\eta)\right)$ to verify that

$$
|q(t)|<\omega(x(t)) \quad \forall t \geq 0 .
$$

Suppose that $t^{\prime}<\infty$. Then $\left\langle x\left(t^{\prime}\right), q\left(t^{\prime}\right)\right\rangle \in \operatorname{bd} \Xi(\mathcal{X}, \omega)$. Since $\Xi(\mathcal{X})$ is closed and $\langle x(t), q(t)\rangle \in \Xi(\mathcal{X})$ on $\left[0, t^{\prime}\right)$, it follows from (13) that $\left\langle x\left(t^{\prime}\right), q\left(t^{\prime}\right)\right\rangle \in \Xi(\mathcal{X}, \omega)$, contradicting the openness of $\Xi(\mathcal{X}, \omega)$. It follows that $t^{\prime}=+\infty$, so the solution $y(t):=y(t, \eta, \mathbf{u}, \mathbf{v})$ of the system (6) maps all of $\mathbf{R}_{\geq 0}$ into $\mathbf{T N}_{\omega} \mathcal{X}$.

Finally, we define a control $\mathbf{w}:[0, \infty) \rightarrow \mathbf{R}^{k}$ by

$$
\mathbf{w}(t)=f_{1}(y(t, \eta, \mathbf{u}, \mathbf{v}), \mathbf{u}(t), \mathbf{v}(t)) .
$$

The control $\mathbf{w}$ cancels the effect of $\phi$ in $\left[8\right.$ for states in $\mathbf{T} \mathbf{N}_{\omega} \mathcal{X}$. In fact,

$$
f_{2}(y(t, \eta, \mathbf{u}, \mathbf{v}), \mathbf{u}(t), \mathbf{v}(t), \mathbf{w}(t)) \equiv f_{1}(y(t, \eta, \mathbf{u}, \mathbf{v}), \mathbf{u}(t), \mathbf{v}(t)),
$$

hence $y(t, \eta, \mathbf{u}, \mathbf{v}) \equiv z(t, \eta, \mathbf{u}, \mathbf{v}, \mathbf{w})$. By our choices of $T_{1}, p_{4}$, and $\mathbf{v}$, we have (a) $x(t)=\pi_{M}(y(t, \eta, \mathbf{u}, \mathbf{v})) \in \mathcal{A}_{1}$ for all $t \geq T_{1}$ and (b) $\left|\pi_{N}(y(t, \eta, \mathbf{u}, \mathbf{v}))\right|<p_{4} / 2 \leq \varepsilon$ for all $t>T_{2}$. It therefore follows that

$$
z(t, \eta, \mathbf{u}, \mathbf{v}, \mathbf{w})=y(t, \eta, \mathbf{u}, \mathbf{v}) \in \mathbf{T N}_{\varepsilon} \mathcal{A}_{1} \subseteq \mathcal{B} \quad \forall t>T
$$

where $T:=\max \left\{T_{1}, T_{2}\right\}$. This shows the asymptotic controllability of $[8$ to our arbitrary neighborhood $\mathcal{B}$ of $\operatorname{clos} \mathbf{T} \mathbf{N}_{\varepsilon} \mathcal{A}$ from any initial value in $\mathbf{T N}_{\omega} \mathcal{X}$. We next show that this controllability property holds from initial values outside $\mathbf{T N}_{\omega} \mathcal{X}$ as well.

Assume then that $z_{0} \notin \mathbf{T N}_{\omega} \mathcal{X}$, so $z_{0} \in C_{\omega}$. We reduce to the case where the initial value is in $\mathbf{T N}_{\omega} \mathcal{X}$. Let $p_{5}=\operatorname{dist}\left(\mathcal{X}, z_{0}\right)$ and let $\eta_{1} \in \mathcal{X}$ be such that $\left|\eta_{1}-z_{0}\right|=p_{5}$. Define $\overline{\mathbf{w}}$ and $z(t)$ by

$$
\overline{\mathbf{w}}(t)=\frac{\eta_{1}-z_{0}}{\left|\eta_{1}-z_{0}\right|} \quad \forall t \geq 0 ; \quad z(t)=z_{0}+t \frac{\eta_{1}-z_{0}}{\left|\eta_{1}-z_{0}\right|}, \quad 0 \leq t \leq \hat{t}:=\inf \left\{t \geq 0: z(t) \in \operatorname{bd} C_{\omega}\right\}
$$

Then $z(t)$ is a solution of $(8)$ starting at $z_{0}$ for any controls $\langle\mathbf{u}, \mathbf{v}\rangle$ and the choice $\mathbf{w}=\overline{\mathbf{w}}$, and $z(t) \in C_{\omega}$ on $[0, \hat{t})$. Also, $0<\hat{t} \leq\left|\eta_{1}-z_{0}\right|$, since if it were the case that $\hat{t}>\left|\eta_{1}-z_{0}\right|$, then setting $t=\left|\eta_{1}-z_{0}\right|$ in (15) would give 
$z(t)=\eta_{1} \in \mathcal{X} \cap C_{\omega}$. This would contradict the fact that $C_{\omega} \subseteq \mathbf{R}^{k} \backslash \mathcal{X}$. We conclude in particular that $\hat{t}<\infty$, so $\eta:=z\left(\hat{t}, z_{0}, \mathbf{u}, \mathbf{v}, \overline{\mathbf{w}}\right) \in \operatorname{bd} C_{\omega} \subseteq \mathbf{T N}_{\omega} \mathcal{X}$. For our precompact open set $\mathcal{B}$, we now construct the controls $\mathbf{u}$ from the controllability of (1), $\mathbf{v}$ as in (11), and $\mathbf{w}$ as in (14), driving this choice of $\eta$ to $\mathcal{B}$. Let $\mathbf{u}^{\sharp}$ and $\mathbf{v}^{\sharp}$ be the concatenations of the zero functions on $[0, \hat{t})$, followed by $\mathbf{u}$ and $\mathbf{v}$, respectively. Let $\mathbf{w}^{\sharp}$ be the concatenation of $\overline{\mathbf{w}}$ on $t \in[0, \hat{t})$ from (15), followed by $\mathbf{w}$ from 14 for $t \geq \hat{t}$. The control vector $\langle\mathbf{u}, \mathbf{v}, \mathbf{w}\rangle$ for $(8)$ drives $\eta$ to $\mathcal{B}$ in time $T$, so $z\left(t, z_{0}, \mathbf{u}^{\sharp}, \mathbf{v}^{\sharp}, \mathbf{w}^{\sharp}\right) \in \mathcal{B}$ for all $t>\hat{t}+T$. Since $T$ and $\hat{t}$ are locally bounded functions of $z_{o}$ and $\mathcal{B}$, we conclude that Conditions 1 (a)-(b) from the GAC definition hold for $(8)$ and the attractor $\operatorname{clos} \mathbf{T N} \mathcal{A}_{\varepsilon} \mathcal{A}$.

To establish Condition 1 (c) of the GAC definition for $\left[8\right.$, fix any precompact open set $E \subseteq \mathbf{R}^{k}$ containing $\operatorname{clos} \mathbf{T N}_{\varepsilon} \mathcal{A}$. We can find an open set $\mathcal{E}_{1} \in \mathcal{P} \mathcal{N}^{\mathcal{A}}$ and $\varepsilon^{\prime}>\varepsilon$ such that $\varepsilon^{\prime}<\omega(x)$ for all $x \in \mathcal{E}_{1}$, and such that

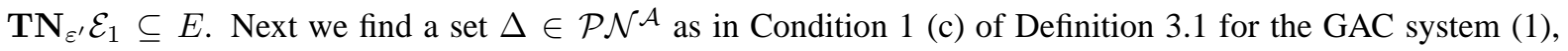
corresponding to $\mathcal{E}_{1}$. It follows that $\operatorname{clos} \mathbf{T} \mathbf{N}_{\varepsilon} \mathcal{A} \subseteq \mathbf{T N}_{\varepsilon^{\prime}} \Delta$. By reducing $\Delta$, we can assume $\varepsilon^{\prime}<\omega(x)$ for all $x \in \Delta$, and therefore $\mathbf{T N}_{\varepsilon^{\prime}} \Delta \subseteq \mathbf{T N}_{\omega} \Delta \subseteq \mathbf{T N}_{\omega} \mathcal{X}$. We show that if $z_{0} \in D:=\mathbf{T N}_{\varepsilon^{\prime}} \Delta$, then $z_{0}$ can be driven to $\mathcal{B}$ using the system $[6$ and the vector of controls $\langle\mathbf{u}, \mathbf{v}\rangle$ as defined above, hence also by the extended system (8), while being kept inside $\mathbf{T N}_{\varepsilon^{\prime}} \mathcal{E}_{1} \subseteq E$ for all $t \geq 0$.

Let $z_{0} \in D$. Since $\pi_{M}\left(z_{o}\right) \in \Delta$, we can arrange (by the choice of $\Delta$ ) that $\mathbf{u}$ is such that $x\left(t, \pi_{M}\left(z_{0}\right), \mathbf{u}\right) \in \mathcal{E}_{1}$ for all $t \geq 0$. Next, we construct $\mathbf{v}$ defined by (11) for the initial state $z_{0} \in \mathbf{T N}_{\varepsilon^{\prime}} \Delta \subseteq \mathbf{T N}_{\omega} \mathcal{X}$. By (i), we know $t \mapsto\left|\pi_{N}\left(y\left(t, z_{0}, \mathbf{u}, \mathbf{v}\right)\right)\right|$ is non-increasing. Thus, for all $t \geq 0$, we get $\left|\pi_{N}\left(y\left(t, z_{0}, \mathbf{u}, \mathbf{v}\right)\right)\right| \leq\left|\pi_{N}\left(z_{0}\right)\right|<\varepsilon^{\prime}$ and therefore also $y\left(t, z_{0}, \mathbf{u}, \mathbf{v}\right) \in \mathbf{T N}_{\varepsilon^{\prime}} \mathcal{E}_{1} \subseteq E$, proving Condition 1 (c) from the GAC definition for system $[8$.

It remains to check that the concatenated controls $\mathbf{u}^{\sharp}, \mathbf{v}^{\sharp}$, and $\mathbf{w}^{\sharp}$ we constructed above satisfy the boundedness requirement from Condition 2 of the GAC definition. That is, we need to check that $\left\|\left\langle\mathbf{u}^{\sharp}, \mathbf{v}^{\sharp}, \mathbf{w}^{\sharp}\right\rangle\right\|$ is a locally bounded function of the initial state $z_{0}$. To do this, first note that the boundedness requirement on $\mathbf{u}^{\sharp}$ is satisfied because (1) is assumed to be GAC to $\mathcal{A}$ on $\mathcal{X}$. Next, $\left\|\mathbf{v}^{\sharp}\right\| \leq p_{2} p_{3} /\left(p_{4} / 4\right)$, and (letting $\mathbf{u}$ be the second part of the concatenation $\mathbf{u}^{\sharp}$ and similarly for $\mathbf{v}$, as before)

$$
\left\|\mathbf{w}^{\sharp}\right\| \leq 1+\operatorname{ess} \sup _{t \geq 0}\left\{\left|f\left(\pi_{M}(y(t, \eta, \mathbf{u}, \mathbf{v})), \mathbf{u}(t)\right)\right|+\left|\pi_{N}(y(t, \eta, \mathbf{u}, \mathbf{v}))\right| \frac{p_{2} p_{3}}{p_{4} / 4}\right\} .
$$

Here $f\left(\pi_{M}(y(t, \eta, \mathbf{u}, \mathbf{v})), \mathbf{u}(t)\right)$ stays bounded because (a) $\pi_{M}(y(t, \eta, \mathbf{u}, \mathbf{v}))=x\left(t, \pi_{M}(\eta), \mathbf{u}\right) \in \bar{B}$ for all $t \geq 0$ and (b) $\bar{B}$ and the $p_{i}$ 's are locally bounded functions of the state $\eta=z\left(\hat{t}, z_{o}, \mathbf{u}^{\sharp}, \mathbf{v}^{\sharp}, \mathbf{w}^{\sharp}\right)$. Also, $\left|\pi_{N}(y(t, \eta, \mathbf{u}, \mathbf{v}))\right|$ 
stays bounded because it decreases from $\left|\pi_{N}(\eta)\right|$. Hence, Condition 2 of the GAC definition holds. This completes the proof of Lemma4.1

Finally, we prove Theorem 2 The preceding argument applied to $\eta \in \operatorname{clos} \mathbf{T} \mathbf{N}_{\varepsilon} \mathcal{A}$ (with $\mathbf{u}$ chosen so that $x\left(t, \pi_{M}(\eta), \mathbf{u}\right) \in \mathcal{A}$ for all $t \geq 0$, which exists by the weak invariance of $\left.\mathcal{A}\right)$ shows that the compact set $\operatorname{clos} \mathbf{T} \mathbf{N}_{\varepsilon} \mathcal{A}$ is weakly invariant for (8). Since (8) is GAC to $\cos \mathbf{T} \mathbf{N}_{\varepsilon} \mathcal{A}$ on $\mathbf{R}^{k}$, the CLSS Theorem (namely, Theorem 1 above) provides an s-stabilizing feedback $K(x)$ for 8 . By Lemma $3.5 \mathcal{X}$ is strongly invariant for $f_{2}$. It follows that the $u$-part of $K(x)$ stabilizes [1]. This establishes Theorem 2

\section{Illustration}

We next illustrate our stabilization approach using the system

$$
\dot{x}=A_{1}(x) u_{1}+A_{2}(x) u_{2} \in T_{x}(\mathcal{X}), \quad x \in \mathcal{X}, \quad u=\left\langle u_{1}, u_{2}\right\rangle \in \mathbf{R}^{2}
$$

evolving on the sphere $\mathcal{X}:=S^{2}=\left\{x \in \mathbf{R}^{3}:|x|=1\right\}$. This simple example will illustrate how to construct stabilizing state feedbacks and Lyapunov functions on smooth manifolds. Even in this simple case, we will see the necessity for using discontinuous stabilizers. Our example is a modified version of the engineering examples in [6]. We choose the attractor $\mathcal{A}=\{ \pm q\}$, where $q=\langle 0,0,1\rangle$, but similar constructions apply for any $q \in S^{2}$. The vector fields $A_{1}$ and $A_{2}$ are chosen as follows. First define $B_{1}(x)=q-(x \cdot q) x$ and $B_{2}(x)=x \times q$, which form an orthogonal basis for the tangent spaces $T_{x}(\mathcal{X})=\operatorname{span}\{x\}^{\perp}$ on $S^{2} \backslash \mathcal{A}$ (in terms of the cross product $\times$, the standard inner product ·, and the orthogonal complement $\perp$ ). Define the geodesic distance $\mathcal{G}$ on $S^{2}$ by

$$
\mathcal{G}\left(x, x^{\prime}\right):=\arccos \left(x \cdot x^{\prime}\right), \text { for } x, x^{\prime} \in S^{2} .
$$

Set $r=\langle 0,1,0\rangle \in S^{2}$ and

$$
V_{q}(x)=\min \{\mathcal{G}(x, \bar{q}): \bar{q} \in\{ \pm q\}\} \text { and } V_{r}(x)=\max \{\mathcal{G}(x, \bar{r}): \bar{r} \in\{ \pm r\}\}, \quad x \in S^{2}
$$

Note the asymmetry between $V_{q}$ and $V_{r}$. Roughly speaking, we use a max in $V_{r}$ to produce a component in our Lyapunov function that penalizes states near $\pm r$ (see (21). Let $M_{1}: S^{2} \rightarrow[0,1]$ be any smooth function satisfying: 
- $M_{1}^{-1}(0)=\left\{x \in S^{2}: \frac{x_{1}}{4} \leq x_{2} \leq \frac{3 x_{1}}{4}\right.$ and $\left.V_{q}(x) \geq \frac{\pi}{4}\right\}$

- $M_{1}^{-1}(1) \supseteq\left\{x \in S^{2}: x_{2} \geq \frac{7 x_{1}}{8}\right.$ or $x_{2} \leq \frac{x_{1}}{8}$ or $\left.V_{q}(x) \leq \frac{\pi}{8}\right\}$

and set

$$
A_{1}(x)=M_{1}(x) B_{1}(x), \quad A_{2}(x)=B_{2}(x)
$$

The factor $M_{1}$ in 18 introduces a set of zeros in $A_{1}$, consisting of a geodesic rectangle covering a part of the equator of $S^{2}$ in the quadrant $Q_{++}:=\left\{x \in S^{2}: x_{1}>0, x_{2}>0\right\}$. In particular, the system (16) is not completely controllable. The fact that $(16)$ is GAC to $\mathcal{A}$ follows because any initial value can be moved to $\mathcal{A}$ along the geodesic direction (i.e., "north" or "south" along a great circle through $\pm q$ ) using the vector field $A_{1}$, possibly by first using $A_{2}$ to move the state "west" out of $M_{1}^{-1}([0,1))$; see below for a precise definition of these stabilizing trajectories. In fact, this global stabilization is done by the discontinuous feedback (20) we construct below. On the other hand, a simple continuous dependence and separation argument (e.g., the argument from the appendix in [19]) shows that the system has no Lipschitz stabilizing state feedback $K(x)$.

The extension of (16) from the Generalized CLSS Theorem amounts to projecting onto the sphere, as follows. The state space $\mathcal{X}$ embeds into $\mathbf{R}^{3}$ by inclusion and $\Xi(\mathcal{X})=\{\langle x, k x\rangle: x \in \mathcal{X}, k \in \mathbf{R}\}$. We can choose $\omega(x) \equiv 1 / 4$ on $\mathcal{X}$. This gives the $\omega$-tube and annular tubular neighborhood

$$
\Xi(\mathcal{X}, \omega)=\left\{\langle x, k x\rangle \in \mathcal{X} \times \mathbf{R}^{3}:|k|<1 / 4\right\}, \quad \mathbf{T N}_{\omega} \mathcal{X}=\theta(\Xi(\mathcal{X}, \omega))=\left\{x \in \mathbf{R}^{3}: 3 / 4<|x|<5 / 4\right\} .
$$

In terms of the projection $\Pi_{s}(y):=y /|y|$ defined on $\mathbf{R}^{3} \backslash\{0\}$, our corresponding system $f_{1}$ on $\mathbf{T} \mathbf{N}_{\omega} \mathcal{X}$ is (see (6))

$$
f_{1}(y, u, v)=M_{1}\left(\Pi_{s}(y)\right)\left(q-\left\{\Pi_{s}(y) \cdot q\right\} \Pi_{s}(y)\right) u_{1}+\left\{\Pi_{s}(y) \times q\right\} u_{2}+\left(y-\Pi_{s}(y)\right) v, \quad\langle u, v\rangle \in \mathbf{R}^{2} \times \mathbf{R},
$$

which we (discontinuously) extend to $\mathbf{R}^{3}$ by setting $f_{1} \equiv 0$ for states outside $\mathbf{T} \mathbf{N}_{\omega} \mathcal{X}$. We next choose

$$
\mathcal{X}^{\sharp}=\left\{z \in \mathbf{R}^{3}: 7 / 8 \leq|z| \leq 9 / 8\right\}, \quad C_{\omega}=\mathbf{R}^{3} \backslash\left\{z \in \mathbf{R}^{3}: 13 / 16 \leq|z| \leq 19 / 16\right\} .
$$

Our corresponding system $f_{2}$ on $\mathbf{R}^{3}$ can then be defined by taking $\phi(z)=\Gamma\left(|z|^{2}\right)$ for any smooth function $\Gamma:[0, \infty) \rightarrow[0,1]$ that satisfies (i) $\Gamma \equiv 1$ on $\left[(7 / 8)^{2},(9 / 8)^{2}\right]$ and (ii) $\Gamma \equiv 0$ outside $\left((13 / 16)^{2},(19 / 16)^{2}\right)$. Since 
$f_{2}$ is GAC to $\mathbf{T N}_{\omega / 2} \mathcal{A}$, there exists a sample stabilizing feedback for $f_{2}$ whose restriction $K(x)$ to $\mathcal{X}$ stabilizes (16) to $\mathcal{A}$. This is the content of our Generalized CLSS Theorem.

The stabilizing feedback $K(x)$ and a corresponding control-Lyapunov function (CLF) can be explicitly constructed by the following variant of the argument from [6] Section 2]. Set $\mathcal{A}^{\sharp}=\{ \pm q, \pm r\} \subseteq S^{2}$. Define

$$
Y_{x}^{\bar{p}}:=\Pi_{s}(\bar{p}-\{\bar{p} \cdot x\} x), \quad \bar{p} \in \mathcal{A}^{\sharp}, x \neq \pm \bar{p} ;
$$

this gives the geodesic direction from $x$ to $\bar{p}$. Note that

$$
A_{1}(x) \cdot Y_{x}^{q} \equiv \sqrt{1-x_{3}^{2}} \text { and } A_{2}(x) \cdot Y_{x}^{q} \equiv 0
$$

Also, $Y_{x}^{-\bar{p}} \equiv-Y_{x}^{\bar{p}}$ for all $\bar{p} \in \mathcal{A}^{\sharp}$. A straightforward calculation (see [6, Lemma 1]) shows that along any (open loop) trajectory of 16 that does not pass through $\mathcal{A}^{\sharp}$, we get

$$
\frac{d}{d t} \mathcal{G}(x, \bar{p})=-\dot{x} \cdot Y_{x}^{\bar{p}}, \quad \bar{p} \in \mathcal{A}^{\sharp}, x \neq \pm \bar{p} .
$$

We show that 16 can be globally stabilized to $\mathcal{A}$ by the (necessarily discontinuous) state feedback

$$
K(x)=\left\{\begin{aligned}
\langle 1,0\rangle, & \text { if } x_{3} \geq 0 \text { and } M_{1}(x)=1 \\
-\langle 1,0\rangle, & \text { if } x_{3}<0 \text { and } M_{1}(x)=1 \\
\langle 0,1\rangle, & \text { if } M_{1}(x)<1
\end{aligned}\right.
$$

when the closed-loop trajectories are defined in the usual non-sampling sense. An easy argument will then show that (20) also sample stabilizes (16). Before presenting our argument, we interpret (20) in terms of the corresponding closed loop (non-sampling) trajectories. For values where $M_{1}(x)=1$, the feedback $K$ drives the state to $\mathcal{A}$ geodesically along a great circle through $\pm q$. On the other hand, any state where $M_{1}(x)<1$ is driven towards $-r$ until the state reaches $M_{1}^{-1}(1)$ and then geodesically to $\mathcal{A}$.

We first analyze the usual non-sampling trajectories of the closed loop system for $K(x)$ which we refer to simply as "closed loop trajectories" in the sequel. The fact that $K(x)$ stabilizes the closed loop trajectories to $\mathcal{A}$ can be verified using the following Lyapunov function construction. In terms of $V_{q}$ and $V_{r}$ in (17), set

$$
V(x)=V_{q}(x)\left[1+V_{r}(x)\right], \quad x \in S^{2} .
$$


Then $V$ is continuous and nonnnegative, and $V$ is null only on $\mathcal{A}$. We will show that $V$ is an integral Lyapunov function for (16) in the sense of [1]; this will imply that $V$ is also a CLF in the usual Dini derivative sense used for example in [7]. ${ }^{2}$ Given a closed loop trajectory $x(t)$, we also let $\dot{V}(x)$ denote the derivative of $t \mapsto V(x(t))$ when it is defined. Along any trajectory $x(t)$ of the closed loop system that remains in $M_{1}^{-1}(1) \backslash \mathcal{A}^{\sharp}$ and that satisfies $x_{2}>0$ and $x_{3}>0$ everywhere, we get $V(x)=\mathcal{G}(x, q)[1+\mathcal{G}(x,-r)]$ and therefore 19 gives

$$
\begin{aligned}
\dot{V}(x) & =-\dot{x} \cdot Y_{x}^{q}[1+\mathcal{G}(x,-r)]-\dot{x} \cdot Y_{x}^{-r} \mathcal{G}(x, q) \\
& =-A_{1}(x) \cdot Y_{x}^{q}[1+\mathcal{G}(x,-r)]+A_{1}(x) \cdot \Pi_{s}(r-\{x \cdot r\} x) \mathcal{G}(x, q) \\
& =-A_{1}(x) \cdot Y_{x}^{q}[1+\mathcal{G}(x,-r)]-\frac{x_{2} x_{3}}{|r-(x \cdot r) x|} \mathcal{G}(x, q) \leq-\sqrt{1-(x \cdot q)^{2}}
\end{aligned}
$$

which is only zero when $x \in \mathcal{A}$. Similar arguments show that

$$
\dot{V}(x) \leq-\sqrt{1-(x \cdot q)^{2}}, \text { if } x_{2} \neq 0, \quad x_{3} \neq 0, \quad M_{1}(x)=1, \text { and } x \notin \mathcal{A} \text {, }
$$

and $\dot{V}(x)=-\left(1+\frac{\pi}{2}\right) \sqrt{1-x_{3}^{2}}$ along trajectories in $\left\{x_{2}=0, x_{3} \neq 0\right\}$. Notice that $\dot{V}$ is continuous along closed loop trajectories in $M_{1}^{-1}(1)$ starting outside $\left\{x_{3}=0\right\}$, and that the closed loop trajectories starting in $M_{1}^{-1}(1)$ with $x_{3}(0)=0$ also satisfy $x_{3}(t) \neq 0$ for all $t>0$. This gives $V(x(t))-V(x(0)) \leq-\int_{0}^{t} \sqrt{1-x_{3}^{2}(s)} d s$ along each closed loop trajectory starting in $M_{1}^{-1}(1)$.

On the other hand, along closed loop trajectories in $M_{1}^{-1}([0,1))$, we know that $V_{r}(x)=\mathcal{G}(x,-r)$, so

$$
\begin{aligned}
\dot{V}(x) & =-\dot{x} \cdot Y_{x}^{ \pm q}[1+\mathcal{G}(x,-r)]-\dot{x} \cdot Y_{x}^{-r} V_{q}(x)=-A_{2}(x) \cdot Y_{x}^{-r} V_{q}(x) \quad\left(\text { since } A_{2}(x) \cdot Y_{x}^{q} \equiv 0\right) \\
& =(x \times q) \cdot \Pi_{s}(r-\{x \cdot r\} x) V_{q}(x)=-\frac{x_{1} V_{q}(x)}{\sqrt{x_{1}^{2}+x_{3}^{2}}}=:-\mu(x)
\end{aligned}
$$

\footnotetext{
${ }^{2} \mathrm{~A}$ control-Lyapunov integral function for 16 and $\mathcal{A}$ is defined to be any continuous function $V: \mathcal{X} \rightarrow[0, \infty)$ for which $V^{-1}(0)=\mathcal{A}$ and for which there exist a constant $N>0$ and $\alpha_{3} \in \mathcal{K}$ satisfying: For each $\xi \in \mathcal{X}$, there exists $u \in \mathcal{U}_{N}$ such that $x(t):=x(t, \xi, u)$ is well defined and satisfies (see [1)

$$
V(x(t))-V(\xi) \leq-\int_{0}^{t} \alpha_{3}\left(|x(s)|_{\mathcal{A}}\right) d s \quad \forall t \geq 0 .
$$

We will verify the decay condition 22] using closed loop trajectories and corresponding feedback controls $u(t)=K(x(t))$ for 16. The inequality [22 then gives the usual Dini derivative Lyapunov decay condition for $V$ (e.g., from [7] 9]) once we divide through by $t$ and pass to the liminf. This last step uses the fact that $t \mapsto \dot{x}(t)=f(x(t), K(x(t)))$ is (right) continuous at $t=0$ for each closed loop trajectory $x(t)$ of 16.
} 
when $x_{3} \neq 0$. Notice that $-\mu$ is bounded above by a negative constant in $M_{1}^{-1}([0,1))$. Also, $\dot{V}(x) \equiv-\pi / 2$ along closed loop trajectories in $M_{1}^{-1}([0,1))$ along $x_{3}=0$. Therefore, reasoning exactly as before gives

$$
V(x(t))-V(x(0)) \leq-\int_{0}^{t} \mu(x(s)) d s
$$

along all closed loop trajectories $x(t)$ remaining in $M_{1}^{-1}([0,1))$. Since $M_{1}^{-1}(1)$ is forward invariant for the closed loop trajectories, it follows that the discontinuous feedback $K(x)$ stabilizes the closed loop trajectories of (16) to $\mathcal{A}$, and that $V$ satisfies the requirements for being a control-Lyapunov (integral) function for (16) and also a CLF for (16) in the usual Dini derivative sense of [7]. That $K(x)$ also sample stabilizes (16) now follows because (a) the sampling and (non-sampling) closed loop trajectories agree for initial points in $M_{1}^{-1}(1)$ and (b) the equality $\dot{V}(x)=-\mu(x)$ holds throughout the quadrant $Q_{++}$if we use the control $u \equiv\langle 0,1\rangle$ at all points in $Q_{++}$. In fact, (a) implies that $K$ sample stabilizes (16) for initial values in $M_{1}^{-1}(1)$ for all partitions $\pi$. Also, (b) implies that $K$ sample stabilizes the dynamic for initial values in $M_{1}^{-1}([0,1))$ when $\overline{\mathbf{d}}(\pi)$ is sufficiently small for the sample control value to switch to $\pm\langle 1,0\rangle$ in $M_{1}^{-1}(1)$ but before the first time the sample trajectory exits $Q_{++}$.

\section{Further Extensions}

We next use our results to establish the input-to-state stabilizability (ISSability) of control affine systems

$$
\dot{x}=h(x)+G(x) u, \quad x \in \mathcal{X}, \quad u \in \mathbf{U}=\mathbf{R}^{m}
$$

evolving on smooth Riemannian manifolds $\mathcal{X}$ relative to actuator errors (but see Remark 6.3 below for an extension to fully nonlinear systems). We assume (25) is GAC to a weakly invariant compact nonempty set $\mathcal{A} \subseteq \mathcal{X}$. In this context, $G(x) u=g_{1}(x) u_{1}+\ldots+g_{m}(x) u_{m}$ for locally Lipschitz vector fields $g_{i}: \mathcal{X} \rightarrow \mathbf{R}$. The stabilizers we construct in this section have the additional desirable feature that they are robust to small observation noise in the controllers. For continuous feedback stabilizers, small observation noise in the controllers can be tolerated. However, since our stabilizing feedback may need to be discontinuous (see Section 1 , such noise terms can have a substantial effect on the dynamics. Therefore, the magnitude of the noise needs to be constrained in terms of the sampling frequency (see [10 11 [16] and Definition 6.2] below). 
To make our ISSability notion precise, we first introduce a Riemannian metric on $\mathcal{X}$ to quantify observation noise and we let $\mathcal{B}_{r}(y)$ denote the corresponding closed ball in $\mathcal{X}$ centered at $y \in \mathcal{X}$ of radius $r$. As before, a feedback for 25 is defined to be any locally bounded function $k: \mathcal{X} \rightarrow \mathbf{U}$. We introduce the set of functions $\mathcal{O}=\{e:[0, \infty) \rightarrow[0, \infty)\}$, which represent the observation errors in our controller; and for each $e \in \mathcal{O}$, we set $\sup (e)=\sup \{e(t): t \geq 0\}$. We use the set of functions $\mathcal{O}(\eta):=\{e \in \mathcal{O}: \sup (e) \leq \eta\}$ for each $\eta>0$. We let Par denote the set of all partitions and

$$
\operatorname{Par}(\delta):=\{\pi \in \operatorname{Par}: \overline{\mathbf{d}}(\pi)<\delta\}
$$

for each $\delta>0$. Our ISSability goal of this section is to find a feedback $k$ so that

$$
\dot{x}(t)=h(x(t))+G(x(t))[k(\eta(t))+\mathbf{u}(t)], \quad \eta(t) \in \mathcal{B}_{e(t)}(x(t))
$$

is input-to-state stable (ISS) for sampling solutions relative to actuator errors $\mathbf{u}$ for small observation errors $e$. The relevant definitions are as follows:

Definition 6.1 Let $k$ be a feedback for [25], $e \in \mathcal{O}, \mathbf{u} \in \mathcal{U}, \xi \in \mathcal{X}$, and $\pi=\left\{t_{i}\right\}_{i \geq 0}$ be any partition of $\mathbf{R}_{\geq 0}$. A $\pi$-solution for (26), the initial state $\xi$, the observation error $e \in \mathcal{O}$, and $\mathbf{u}$ is defined to be any continuous function $x(\cdot)$ obtained by recursively choosing any $\eta\left(t_{i}\right) \in \mathcal{B}_{e\left(t_{i}\right)}\left(x\left(t_{i}\right)\right)$ and then solving

$$
\dot{x}(t)=h(x(t))+G(x(t))\left[k\left(\eta\left(t_{i}\right)\right)+\mathbf{u}(t)\right]
$$

from the initial time $t=t_{i}$ up to time

$$
s_{i}=\max \left\{t_{i}, \sup \left\{s \in\left[t_{i}, t_{i+1}\right]: x(\cdot) \text { is defined on }\left[t_{i}, s\right)\right\}\right\},
$$

where $x(0)=\xi \cdot{ }^{3}$ The domain of $x(\cdot)$ is $\left[0, t_{\max }\right)$, where $t_{\max }=\inf \left\{s_{i}: s_{i}<t_{i+1}\right\}$. When $t_{\max }=+\infty$, we call $x(t)$ well defined.

Definition 6.2 Let $k$ be a feedback for (25). We say $k$ renders (25) sample-input-to-state stable (s-ISS) to $\mathcal{A}$ provided for each $\mathcal{R}_{o} \in \mathcal{P} \mathcal{N}^{\mathcal{A}}$ and each $N>0$, there exists $\mathcal{R}_{1}=\mathcal{R}_{1}(N) \in \mathcal{P} \mathcal{N}^{\mathcal{R}_{0}}$ such that:

\footnotetext{
${ }^{3}$ As before, the continuity requirement for $x(\cdot)$ stipulates that the final value on the previous subinterval is used as the initial value at the next subinterval. Also, the $t_{i}$ argument of the max 27] allows the possibility that $x(t)$ is not defined at all on $\left[t_{i}, t_{i+1}\right]$ (see Section 2 .
} 
1. For each $\mathcal{R}_{2}, \mathcal{R}_{3} \in \mathcal{P} \mathcal{N}^{\mathcal{R}_{1}}$ with $\mathcal{R}_{2} \subseteq \mathcal{R}_{3}$, there exist $\mathcal{M}=\mathcal{M}\left(\mathcal{R}_{3}\right) \subseteq \mathcal{X}$ and positive numbers $\delta$, $T$, and $\kappa$ (depending on $\mathcal{R}_{2}$ and $\mathcal{R}_{3}$ ) such that if $\pi \in \operatorname{Par}(\delta), \xi \in \mathcal{R}_{3}, \mathbf{u} \in \mathcal{U}_{N}$, and $e \in \mathcal{O}(\kappa \underline{\mathbf{d}}(\pi))$, then the corresponding $\pi$-solutions $x(t)$ for (26) starting at $\xi$ are all well defined and satisfy (a) $x(t) \in \mathcal{R}_{2}$ for all $t \geq T$ and (b) $x(t) \in \mathcal{M}$ for all $t \geq 0$.

2. For each $\mathcal{E} \in \mathcal{P} \mathcal{N}^{\mathcal{R}_{1}}$, there exists $D \in \mathcal{P N}^{\mathcal{R}_{1}}$ such that if the set $\mathcal{R}_{3}$ in 1 . is a subset of $D$, then the set $\mathcal{M}$ in 1 . can be chosen to be a subset of $\mathcal{E}$.

and for each $\mathcal{R}_{0} \in \mathcal{P} \mathcal{N}^{\mathcal{A}}$, there exists $N=N\left(\mathcal{R}_{0}\right)>0$ such that 1.-2. hold with the choices $N=N\left(\mathcal{R}_{0}\right)$ and $\mathcal{R}_{1}=\mathcal{R}_{0}$. In this case, we also say (26) is ISS for sampling solutions and that 25 is ISSable.

The preceding definition requires that the sampling be done quickly enough so that $\pi \in \operatorname{Par}(\delta)$, but not so quickly that $\sup (e)>\kappa \underline{\mathbf{d}}(\pi)$. When $e \equiv 0$, the condition on $\underline{\mathbf{d}}(\pi)$ in Definition 6.2 is not needed. For $\mathcal{X}=\mathbf{R}^{n}$, one can easily check that if (25) is sampling ISS in the sense defined in [10, 11] using some feedback $k$, then it is also ISSable in the sense of Definition 6.2 with the same feedback $k$. For any compact nonempty weakly invariant set $\mathcal{A}$ for 25 , we then have:

Theorem 3 If (25) is GAC to $\mathcal{A}$, then there exists a feedback $k(x)$ rendering (25) s-ISS to $\mathcal{A}$.

Proof. We indicate the changes needed in the proof of Theorem 2 As before, we first extend the dynamics

$$
f(x, u)=h(x)+G(x) u
$$

to a dynamics (8) defined on all of $\mathbf{R}^{k}$ that is GAC to $\cos \mathbf{T} \mathbf{N}_{\varepsilon} \mathcal{A}$. By [9] Theorem 3.2], this extended dynamics admits a locally Lipschitz control-Lyapunov function (CLF) $V$; see [16] for background on CLFs. Using the argument from [13, Section 5], we can transform $V$ into a (locally) semiconcave CLF for [8) on $\mathbf{R}^{k} \backslash \operatorname{clos} \mathbf{T} \mathbf{N}_{\varepsilon} \mathcal{A}$. In [10], it was shown that control affine systems that are GAC to $\{0\}$ on $\mathbf{R}^{k}$ admit (possibly discontinuous) feedbacks for which the corresponding closed loop systems are sampling ISS to $\{0\}$. Since $[\mathbb{8}$ is again control affine, a slight variant of the argument from [10 Section 3] provides a feedback $K(x)$ rendering [8] s-ISS to $\operatorname{clos} \mathbf{T N}_{\varepsilon} \mathcal{A}$. Applying Lemma 3.5 as before, we conclude that the $u$-part of $K(x)$ renders 25 s-ISS to $\mathcal{A}$. 
Remark 6.3 The preceding theorem can be extended to cover fully nonlinear systems 1 on $\mathcal{X} \times \mathbf{R}^{m}$ if we reinterpret s-ISS in the following more general sense: A feedback $k$ renders (1) s-ISS to $\mathcal{A}$ in the weak sense provided there exists a smooth everywhere invertible matrix valued function $G: \mathcal{X} \rightarrow \mathbf{R}^{m \times m}$ such that

$$
\dot{x}=f(x, k(x)+G(x) u)
$$

is s-ISS to $\mathcal{A}$. The s-ISS property for 28 is defined by taking $e \equiv 0$ in Definition 6.2 and the $\pi$-solutions of 28 are defined by recursively solving

$$
\dot{x}(t)=f\left(x(t), k\left(x\left(t_{i}\right)\right)+G(x(t)) \mathbf{u}(t)\right)
$$

on successive intervals $\left[t_{i}, t_{i+1}\right]$ of the partition $\pi=\left\{t_{i}\right\}_{i \geq 0}$ and proceeding as in Definition 6.1 with $e \equiv 0$ (see [10] for details). In particular, the sampling is only done in the (possibly discontinuous) controller $k(x)$. We can then prove the following for any smooth manifold $\mathcal{X}$ and $\mathbf{U}=\mathbf{R}^{m}$ : If (I) is GAC to a compact, nonempty, weakly invariant set $\mathcal{A}$, then there exists a feedback $k(x)$ rendering (1) $s$-ISS to $\mathcal{A}$ in the weak sense. The proof combines the arguments from [10 Section 5] with our proof of Theorem [3] and is left to the reader.

Remark 6.4 As we noted in the introduction, the GAC system (1) will not in general admit a continuous stabilizing state feedback. However, by [8], the system (1) is stabilizable by a continuous time varying feedback $u=k(t, x)$ if it is completely controllable and drift-free (the latter condition being the requirement that $f(x, 0) \equiv 0)$. In engineering applications, feedback laws are usually implemented via sampling. This motivated our construction of discontinuous state stabilizers $u=k(x)$ which we implemented using CLSS solutions. Yet another approach to stabilizing (1) is to look for a dynamic stabilizer. This means finding a locally Lipschitz regulator dynamic $\dot{z}=A(z, x)$ and a locally Lipschitz function $k(z, x)$ such that the interconnected system

$$
\dot{x}=f(x, k(z, x)), \quad \dot{z}=A(z, x)
$$

is globally asymptotically stable. See [15] for an extensive discussion of dynamic stabilizers for linear systems.

On the other hand, it turns out that a dynamic feedback for (1) may fail to exist, even if the system is completely controllable. An example from [19] where this occurs is

$$
\dot{x}=f(x, u)=\left[\begin{array}{l}
\left(4-x_{2}^{2}\right) u_{2}^{2} \\
e^{-x_{1}}+x_{2}-2 e^{-x_{1}} \sin ^{2}\left(u_{1}\right)
\end{array}\right], x \in \mathbf{R}^{2}, u \in \mathbf{R}^{2} .
$$


The fact that 29 is completely controllable (and therefore GAC to $\mathcal{A}=\{0\}$ ) was shown in the appendix of [19], where it is also shown that it is impossible to choose paths converging to the origin in such a manner that this selection is continuous in the initial states. Since the flow map of any dynamic stabilizer would give a continuous choice of paths converging to the origin, no dynamic stabilizer for the system can exist, even if we drop the requirement that the state of the regulator converges to zero. In particular, we see that (29) cannot admit a continuous time varying feedback $u=k(t, x)$. This does not contradict the existence results [8] for time varying feedbacks since in this case, the system has drift.

Remark 6.5 The feedback construction [10] used to prove Theorem 3 proceeds by first finding a semiconcave control-Lyapunov function (CLF) for the system and then adapting the feedback design from [14] to allow nonsmooth CLFs, observation noise, and discontinuous feedback. Semiconcave CLFs are known to exist for all (locally Lipschitz) GAC systems on Euclidean space and all compact nonempty weakly invariant attractors $\mathcal{A}$, by arguments from [13]. The semiconcavity property is intermediate between $C^{1}$ and local Lipschitzness. On the other hand, GAC systems will not in general admit smooth CLFs since their existence would imply the existence of continuous stabilizers $k(x)$, which we know not to be the case in general (see [5] 16]).

For a very different approach to ISS on manifolds (based on density functions) that gives rise to a sufficient condition for ISS-like behavior from almost all initial values, see [2]. The main ISS-like condition in [2] states: For a given Riemannian manifold $\mathcal{X}$ and a compact weakly invariant set $\mathcal{A} \subseteq \mathcal{X}$ for $\mathbb{1}$, we say that $\mathbb{1}$ is weakly almost ISS to $\mathcal{A}$ provided (i) $\mathcal{A}$ is locally asymptotically stable for the system and (ii) there exists $\gamma \in \mathcal{K}$ such that

$$
\forall u \in \mathcal{U}, \quad \exists \mathcal{Z}_{u} \in \operatorname{Null}(\mathcal{X}) \text { s.t. } \forall \xi \in \mathcal{X} \backslash \mathcal{Z}_{u}, \liminf _{t \rightarrow+\infty}|x(t, \xi, u)|_{\mathcal{A}} \leq \gamma(\|u\|)
$$

where $\operatorname{Null}(\mathcal{X})$ is the set of subsets of $\mathcal{X}$ of measure zero and $|\cdot|_{\mathcal{A}}$ denotes the distance to $\mathcal{A}$. This condition differs from our ISS requirement mainly in its allowance of a null set of states that are not necessarily stabilized and in its use of Carathéodory solutions. An alternative and more intrinsic approach to feedback stabilization on manifolds would involve generalizing the concepts of set valued differentials and semiconcave CLFs to manifolds and providing direct feedback constructions without first embedding into $\mathbf{R}^{k}$. We provided a first result in this direction in Section 5 above. We leave the development of this more intrinsic approach for another paper. 


\section{Acknowledgments}

M. Malisoff was supported by Louisiana Board of Regents Contract LEQSF(2003-06)-RD-A-12. Part of the work

of M. Krichman was carried out while this author was a Research Assistant at Rutgers University. Krichman thanks Felipe M. Pait for helpful comments. E. Sontag was supported by NSF Grant CCR-0206789. 


\section{References}

[1] F. Albertini and E. Sontag, "Continuous control-Lyapunov functions for asymptotically controllable time varying-systems," International J. Control 72(1999):1630-1641.

[2] D. Angeli, “An almost global notion of input-to-state stability," IEEE Transactions Automat. Control 49(2004):866-874.

[3] W. Boothby, An Introduction to Differentiable Manifolds and Riemannian Geometry. Revised Second Edition, Academic Press, New York, 2003.

[4] G. Bredon, Topology and Geometry. Graduate Texts in Mathematics, 139. Springer-Verlag, New York, 1993.

[5] R. Brockett, "Asymptotic stability and feedback stabilization," in Differential Geometric Control Theory, R. Brockett, R. Millman, and H. Sussmann, Eds., Birkhäuser, Boston, 1983, pp. 181-191.

[6] F. Bullo, R. Murray, and A. Sarti, "Control on the sphere and reduced attitude stabilization," CDS Technical Report 95-005, and Proc. Nonlinear Control Systems Design (NOLCOS), Tahoe City, CA, June 1995, pp. 495-501. Available at http : //www.cds.caltech.edu/ murray/papers/1995q_bm95a-cds.html.

[7] F. Clarke, Y. Ledyaev, E. Sontag, and A. Subbotin, "Asymptotic controllability implies feedback stabilization," IEEE Transactions Automat. Control 42(1997):1394-1407.

[8] J-M. Coron, "Global asymptotic stabilization for controllable systems without drift," Math. Control Signals Systems 5(1992):295-312.

[9] C. Kellett and A. Teel, "Weak converse Lyapunov function theorems and control-Lyapunov functions," SIAM J. Control Optim. 42(2004):1934-1959.

[10] M. Malisoff, L. Rifford, and E. Sontag, "Global asymptotic controllability implies input-to-state stabilization," SIAM J. Control Optim. 42(2004):2221-2238. 
[11] M. Malisoff and E. Sontag, "Asymptotic controllability and input-to-state stabilization: The effect of actuator errors," in Optimal Control, Stabilization, and Nonsmooth Analysis, M. de Queiroz, M. Malisoff, and P. Wolenski, Eds., Lecture Notes in Control and Information Sciences Vol. 301, Springer-Verlag, New York, 2004, pp. 155-171.

[12] J. Milnor, "Differential topology," in 1964 Lectures on Modern Mathematics, Vol II. Wiley, New York, 1964, pp. 165-183.

[13] L. Rifford, "Existence of Lipschitz and semiconcave control-Lyapunov functions," SIAM J. Control Optim. 39(2000):1043-1064.

[14] E. Sontag, "Smooth stabilization implies coprime factorization," IEEE Transactions Automat. Control 34(1989):435-443.

[15] E. Sontag, Mathematical Control Theory. Deterministic Finite-Dimensional Systems. Second Edition. Texts in Applied Mathematics 6. Springer-Verlag, New York, 1998.

[16] E. Sontag, "Stability and stabilization: Discontinuities and the effect of disturbances," in Nonlinear Analysis, Differential Equations, and Control, F. Clarke and R. Stern, Eds., Kluwer, Dordrecht, The Netherlands, 1999, pp. 551-598.

[17] E. Sontag, "Clocks and insensitivity to small measurement errors," ESAIM Control Optim. Calc. Var. 4(1999):537-557.

[18] E. Sontag and H. Sussmann, "Remarks on continuous feedback," in Proceedings of the IEEE Conference on Decision and Control (Albequerque, NM, December 1980), pp. 916-921.

[19] H. Sussmann, “Subanalytic sets and feedback control," J. Differential Equations 31(1979):31-52. 